\title{
SOCIAL NETWORKING AND THE EXCHANGE OF INFORMATION
}

\author{
Erin K. Wise, Texas A\&M University - Kingsville, erin.wise.tx@gmail.com \\ Jack D. Shorter, Texas A\&M University - Kingsville, jack.shorter@tamuk.edu
}

\begin{abstract}
Social media has become a central point of a person's daily life for many people around the world with the ability to be connected to these sites through access to cellphones, tablets, and computers. That being said, the use of information that is supplied to anyone and everyone on a social media site creates major concerns about individual privacy and concerns about how this information can and is used to attack and destroy lives. The ease of sharing information has allowed people to keep in contact with friends and family and keep them updated on life changes, views of various subjects, collaborate on projects, and much more. It has also made it possible for groups or individuals who do not like you or your beliefs to use this information to harm you in ways never before possible.
\end{abstract}

Keywords: Social Networking, Social Media, Internet, Privacy, Identity Theft

\section{INTRODUCTION}

There are many wonderful things about our modern society, with Social Media being one of those wonderful uses of technology. However, it should be mentioned that technology can be used for both good and bad purposes. If you have ever had aspirations of becoming the President of the United States, you better thing long and hard about what you believe should be posted on your social networking sites. In an era where political campaigns are not about issues, but about how to destroy your opponents good name, anything posted on a social media site is fodder for anyone who supports your opponent. Through social media, people are allowed to quickly share and exchange information with friends, family, and even complete strangers through the "posting" of URLs, images, or statuses on personal pages or through private messaging featured on social media sites. The ease of sharing information has allowed people to keep in contact with friends and family and keep them updated on life changes, views of various subjects, collaborate on projects, and much more. This is all very exciting and wonderful. But, your privacy is compromised by any post at any time on this medium. Information on social media sites makes it much easier to become a victim of identity theft, cyber bullying, and even character assassination. Information that is placed on social media sites can also be stolen or restrictions can be put in place as to what sites can be accessed by members of the public and what can be posted on these sites.

\section{Defining Social Media}

"Social media is the collective of online communications channels dedicated to community-based input, interaction, content-sharing and collaboration." [19] From this definition it can be concluded that sites such as wikis, blogs, forums, and both social and professional networking sites are considered social media. Each of these different types of social media has their own features that differentiate them from one another while still having many, if not all, the features given in the definition.

\section{The Beginnings of Social Media and the Information Exchange}

Social media as described by the above definition began in 1978 with the creation of the bulletin board system (BBS) by Ward Christensen and Randy Suess to inform friends of meetings, announcements, and share information through postings. This was the beginning of virtual communities. These bulletin boards also introduced trolling and flame wars. [4] In 1979, "Usenet allowed users to communicate through a virtual newsletter."[9] Usenet was created by Duke University graduate students, Tom Truscott and Jim Ellis, and Steve Bellovin, a student at University of North Carolina. The vision was to create software that would allow messaging between department members, along with connecting to other universities, which was limited to phone calls and postal newsletters at that time. Duke University and University of North Carolina became the first two hosts for Usenet. The software was compiled for formal distribution and had its name changed to "A News" by Steve Daniel. It was made available to interested systems administrators in 1980 at the Usenix Conference. Because the capabilities of A News were quickly 
outgrown by its network, "B News" was developed and released in 1981. [26] It was with the services that came with America Online (AOL) that became the precursor to modern social networks with its member-created communities and viewable member profiles. [7]

\section{Modern Social Media}

Social networking that resembles what people know today began to appear in 1994 when Geocities was created. Users were able to create websites and sort them into "cities" based on their site content. TheGlobe.com followed a year later when they launched publically. TheGlobe.com allowed users to interact with other users that had similar interests and hobbies and to publish their own content. AOL continued to lead the early years of modern social media with its AOL Messenger that allowed members to write biographies about themselves and share other details that they wished to. AOL Messenger also included members to search for the profiles of other members. [1]

Early social networking sites like Classmates began to appear. Many of these early social networking sites were for dating purposes, but others had specific niches. Classmates would allow its users to find and reconnect with old classmates from school. It continues to still have 40,000,000 registered members as of 2010. SixDegrees.com was another early social networking site, but did not reach the same success that Classmates did. SixDegrees.com allowed users to create profiles and groups and search for and invite friends. Because of the frequent member drives, it didn't become as popular as other social networking sites of the time, due to people considering that the membership invitations were spam. [1]

Friendster was started in 2002 and was very successful, and would go on to inspire Myspace and become "a blueprint for the modern-day social network." [24] In 2003, Myspace was started. It was created by several eUniverse employees who had Friendster Accounts looking to expand upon the potential of the former social networking site's features. Myspace's effect on the social media was that it "significant influence on pop culture and music and created a gaming platform that launched the successes of Zynga and Rock You, among others. The site also started the trend of creating unique URLs for companies and artists." [17]

Social media has evolved from simple bulletin board systems and simple interactions between users to "websites and applications dedicated to forums, microblogging, social networking, social bookmarking, social curation, and wikis. "[19]

\section{Forums}

"An Internet forum, or message board, is an online discussion site where people can hold conversations in the form of posted messages. They differ from chat rooms in that messages are at least temporarily archived. Also, depending on the access level of a user or the forum set-up, a posted message might need to be approved by a moderator before it becomes visible. Forums have a specific set of jargon associated with them; e.g. a single conversation is called a "thread", or topic."[11]

A discussion forum is hierarchical or tree-like in structure: a forum can contain a number of sub-forums, each of which may have several topics. Within a forum's topic, each new discussion started is called a thread, and can be replied to by as many people as so wish. Depending on the forum's settings, users can be anonymous or have to register with the forum and then subsequently $\log$ in in order to post messages. On most forums, users do not have to $\log$ in to read existing messages." [11]

Forums allow users to share information amongst not only other members of the forum, but also if it's an open forum, among all visitors to the forums. In addition, most forums have ways for members to contact one another or simply share information about them in a personal bio.

\section{Microblogs}

"Microblogging is a broadcast medium that exists in the form of blogging. A microblog differs from a traditional blog in that its content is typically smaller in both actual and aggregated file size. Microblogs "allow users to exchange small elements of content such as short sentences, individual images, or video links". [15] "These small 
messages are sometimes called micro posts. As with traditional blogging, micro bloggers post about topics ranging from the simple, such as "what am I doing right now," to the thematic, such as "sports cars." Commercial microblogs also exist to promote websites, services and products, and to promote collaboration within an organization. Some microblogging services offer features such as privacy settings, which allow users to control who can read their microblogs, or alternative ways of publishing entries besides the web-based interface. These may include text messaging, instant messaging, E-mail, digital audio or digital video."[15]

An example of how microblogging can be used to share information is in the case of milk powder companies using the microblogging site Weibo. "The stepped-up monitoring of the microblogging site Weibo and local online forums reflects the outsize role the social media play in China, where access to information is restricted. Chatter about food safety scares spreads with lightning speed on Weibo, so companies are learning to keep constant track of their online brand reputations."[12] The sharing between users of these social media sites concerning milk powder companies, such as which were being recommended as safe after the pricing investigation and food safety scare. It allowed companies to track their reputation. [12]

\section{Social and Professional Networking}

"A social networking service is a platform to build social networks or social relations among people who, for example, share interests, activities, backgrounds, or real-life connections. A social network service consists of a representation of each user (often a profile), his/her social links, and a variety of additional services. Most social network services are web-based and provide means for users to interact over the Internet, such as e-mail and instant messaging. Online community services are sometimes considered as a social network service, though in a broader sense, social network service usually means an individual-centered service whereas online community services are group-centered. Social networking sites allow users to share ideas, pictures, posts, activities, events, and interests with people in their network."[23]

Social and professional networking sites allow users to keep in contact or reconnect with people they have met, meet new people, and share information through status updates, news feeds, and upload. For many users, a social networking site, such as Facebook, allows members to keep in contact with family and friends who they might rarely see or to keep them updated on activities happening in their lives.

\section{Social Bookmarking}

"A social bookmarking service is a centralized online service which enables users to add, annotate, edit, and share bookmarks of web documents. Many online bookmark management services have launched since 1996; Delicious, founded in 2003, popularized the terms "social bookmarking" and "tagging". Tagging is a significant feature of social bookmarking systems, enabling users to organize their bookmarks in flexible ways and develop shared vocabularies known as folksonomies."[22] Social bookmarking allows users to "bookmark" and share web pages, images, and documents found online. An example is of users finding a funny image and then adding or bookmarking it on a website such as Pinterest before sharing it with their friends.

\section{Social Curation}

"Social curation is collaborative sharing of Web content organized around one or more particular themes or topics. Among the oldest social curation sites are Digg and Reddit. Both of those sites allow users to suggest links to articles and allow other readers to give approval - on Digg, for example, by clicking a "thumbs up" icon. Higher approval ratings mean that a story will appear more prominently. Delicious, another long-standing social curation site is dedicated to social bookmarking: Users save and share links to websites of interest, arranged according to topics. A newer social curation site, Pinterest, is dedicated to images." [18] A website like Pinterest allows its users to not only bookmark images, but also create personal boards to hold images that fit into one category together as per the user's preferences. For example, many users on Pinterest have "Pin Boards" that they place various recipes that other users have "pinned" and then share those pins on their boards with their friends. 
Wikis

"A wiki is usually a web application which allows people to add, modify, or delete content in collaboration with others. Text is usually written using a simplified markup language or a rich-text editor. While a wiki is a type of content management system, it differs from a blog or most other such systems in that the content is created without any defined owner or leader, and wikis have little implicit structure, allowing structure to emerge according to the needs of the users. The encyclopedia project Wikipedia is the most popular wiki on the public web in terms of page views, but there are many sites running many different kinds of wiki software. Wikis can serve many different purposes both public and private, including knowledge management, notetaking, community websites and intranets. Some permit control over different functions (levels of access). For example, editing rights may permit changing, adding or removing material. Others may permit access without enforcing access control. Other rules may also be imposed to organize content. Ward Cunningham, the developer of the first wiki software, WikiWikiWeb, originally described it as "the simplest online database that could possibly work"."[28]

Wikis can be found anywhere and on almost any subject. Not only is there the large wiki encyclopedia Wikipedia, but there are also smaller wikis for book series, television shows, movies, and so on. These wikis allow visitors to look up user added and edited information on the subject.

\section{Downsides of Social Media Concerning the Information Exchange}

The restriction of information being shared has become a more rampant issue, especially overseas in countries such as China. For example, according to a New York Times article, "complaints against senior or national-level officials remain taboo and are actively scoured from the Internet by government censors and employees of the companies that operate online forums." [8] These restrictions not only result in the censorship of the information being shared amongst users of social media communities, but can lead to detainment. From a New York Times article concerning the Chinese government, "The government has been cracking down on social media activists who have exposed corruption or stirred up interest in social issues. Some prominent activists have been detained, including a journalist and people working at public relations firms." [2]

The power behind social media and the information that its users are sharing has become something that made governments worry. "Because microblogs have grown into a powerful social force that has led to the downfall of some prominent government officials, analysts say the authorities are determined to put stricter controls in place and have pressed Internet companies to censor more content." [2]

Another example of a government restricting the sharing of information is the Vietnamese government where Dinh Nhat Uy was convicted of abusing democratic freedoms when he was posting criticisms of the government and state-owned companies. "A law took effect in September banning the sharing of news and content critical of the government on social media networks. No one has yet been charged under that law, which given the volume of content carried on Facebook would appear to be hard to enforce. The government attempts to block some websites, but interference is rare on Facebook."[27]

However, sometimes the restrictions are not only made by the governments of countries where users are located. In cases, those restrictions are made by the websites themselves. "How much free speech is Facebook willing to allow? That seems to depend on what kind of speech it is. Videos of people being beheaded appear to cross a linealthough that hasn't always been the case- but other equally violent imagery continues to circulate freely on the network. Photos of women breast-feeding, however, are routinely removed, as are posts by dissident groups in a number of countries, often without explanation." "No matter how you slice it, this puts Facebook into the thick of an editorial decision - and not an easy one, either. Now Facebook no longer looks only at content to determine whether to remove a post, but it also considers the context of the sharing, including the language and other behavior of users. That's a much harder decision, and one that may come back to bite the company, especially given its somewhat contradictory decisions in other cases." [10]

When users sign up for social media sites, they assume that the information that they put up will be only seen by people they allow it to be seen by. This is not always true, through more open default privacy settings and government surveillance. Users trust these sites to keep their information private and safe, and have found out that 


\section{Issues in Information Systems \\ Volume 15, Issue II, pp. 103-109, 2014}

this is not always the case. "The Internet industry, having nudged consumers to share heaps of information about themselves, has built a trove of personal data for government agencies to mine - erecting, perhaps unintentionally, what Alessandro Acquisti, a Carnegie Mellon University behavioral economist, calls "the de facto infrastructure of surveillance."'”[20]

A lot of changes have also been made to the privacy settings more open by default, but allow more options for users to select what they wish to have seen by certain users. For example, Facebook allows its users to keep from being searched for by name in the search bar, however, in December, this feature will be removed for all users, and has already been removed for users that were not using the Timeline feature. [25] In addition, more recently, Facebook has changed its privacy settings concerning teenage users. "Facebook is relaxing its rules for teenagers. The 13- to 17-year-old set now has the option to share photos, updates and comments with the general public on Facebook. That means strangers, and companies collecting data for advertisers and marketing companies, will be able to see select posts. Teenagers will also be able to turn on the Follow feature for their profiles, which would allow anyone they're not friends with to see their public posts in the main news feed."[13] However, Facebook is not the only social media website that allows teenagers to share information publically. "Other social networks such as Twitter, Tumblr and Last.fm don't prevent teens from posting publicly.” [13]

Hacking and stolen information is another issue that has arrived with the heavy use of social media and the amount of information that is being shared across it. For example, the Syrian Electronic Army recently were able to compromise the shortened URLs in tweets that were sent from the president's twitter account and posted to his Facebook account that instead of linking to the donation site instead was redirected to the SEA's site. [16] Another example was in 2011, when "Hackers have broken into the popular South Korean websites Nate and Cyworld earlier this week, and stole information about 35 million social networking users. Names, email addresses, phone numbers and resident registration numbers of users are said to have been compromised." [3]

Along with hacking and stolen information, there has also been the issue of fake accounts and stolen identities, such as in the case of Dr. Jubal Yennie. "The Twitter account was created with the name and photo of Dr. Jubal Yennie, director of the Sullivan County school district. The account has since been deleted but the tweets sent in Yennie's name were reported to be of an embarrassing nature and not appropriate for a school administrator." The fake account was created by Ira Trey Quesenberry III, an 18-year-old student at Sullivan Central High School, and resulted in an identity theft charge. [21]

\section{CONCLUSIONS}

The human species is a social species. One of the major reasons for the computer and eventually the Internet itself was our need to stay in contact with each other. Our curiosity demands it. The positive uses of social media sites on the Internet play into our need to stay connected. Social media has come a long way since its origins as a simple bulletin board system used to keep members updated on meetings, announcements, and information.

Social media juggernauts such as Facebook, Twitter, and YouTube allow users to find one another, keep friends, family, and acquaintances updated, share videos, and much more. However, with the increase in information that is being shared through social mediums, there is a vastly increased risk associated with it. Information loss to hackers, scammers, and phishing attempts, as well as, being monitored by our own government agencies is a real threat to our freedoms.

What can be done to lesson or negate the harmful use of information supplied on the Internet through social networking sites? What can be done to lesson or negate the impact of Identity Theft? What can be done to lesson or negate the impact of cyber bullying? What can be done to lesson or negate the impact of using this information, or misinformation to assassinate ones character? These are questions that beg for answers. These questions also create an opportunity for academic researchers' to explore the myriad ways to tackle these negative uses of information currently posted on social networking sites around the world. What can be done?

We believe that consumers of social networking need to take a much more proactive approach to their privacy and security requirements. The federal government can create laws to protect us from identity theft, cyber bullying, and character assassination. But individuals need to educate themselves about these problems and how they can protect 
themselves and their children from them. Keeping track of your credit rating, your child's Internet use, and googling yourself every once in a while to see what is out there about you on the Internet, are just a few things that individuals can do to protect themselves from social networking negative consequences.

Social media has been around for a long time. The uses and abuses of social media permeate our society now and will continue to in the future. The Internet has changed our world. Lack of privacy is not going away. Social media is an outgrowth of the human species' need to be social. We are a social animal to a fault. This has made it possible for us to conquer the world. However, our tenure on this planet is tenuous. A simple loss of the power grid would throw us back into the 1800 's. And without our technology a large part of the human race could not survive.

\section{REFERENCES}

1. Arandilla, R. (2010). A History You Will Love: How Online Social Networking Began. Retrieved from http://www.1stwebdesigner.com/design/history-social-networking/

2. Barboza, D. (2013, August 25). Chinese-American Commentator and Investor Is Arrested in Beijing. Retrieved from http://www.nytimes.com/2013/08/26/world/asia/chinese-american-commentator-and-investor-isarrested-in-beijing.html? $\mathrm{r}=0$

3. Cluley, G. (2011, July 28). Data stolen from 35 million South Korean social networking users. Retrieved from http://nakedsecurity.sophos.com/2011/07/28/data-stolen-from-35-million-south-korean-social-networkingusers/

4. Curtis, A. (2013). The Brief History of Social Media. Retrieved from http://www.uncp.edu/home/acurtis/ NewMedia/SocialMedia/SocialMediaHistory.html

5. Encyclopedia Britanica Inc. (2012, September 5). computer programming language. Retrieved October 28, 2012, from Encylopdia Britanica: http://www.britannica.com/EBchecked/topic/130670/computer-programminglanguage

6. Encyclopedia Britanica Inc. (2008, October 2008). machine language. Retrieved October 28, 2012, from

Encyclopedia Britanica: http://www.britannica.com/EBchecked/topic/354646/machine-language

7. Goble, G. (2012, September 6). The History of Social Media. Retrieved from http://www.digitaltrends.com/features/the-history-of-social-networking/

8. Gough, N. (2013, September 05). Chinese Official, a Symbol of Greed and Corruption, Is Sentenced. Retrieved from http://www.nytimes.com/2013/09/06/world/asia/yang-dacai-is-sentenced-in-china.html

9. Hendricks, D. (2013, May 8). The Complete History of Social Media: Then and Now. Retrieved from http://smallbiztrends.com/2013/05/the-complete-history-of-social-media-infographic.html

10. Ingram, M. (2013, October 24). Facebook's Standards Problem. Retrieved from http://www.businessweek.com/articles/2013-10-24/facebooks-flip-flops-on-free-speech-may-backfire

11. Internet forum - Wikipedia, the free encyclopedia. (2013, October 19). Retrieved from http://en.wikipedia.org/wiki/Internet_forum

12. Jourdan, A. (2013, August 26). Milk Powder Companies Tune In to Chinese Social Media Reports. Retrieved From http://www.nytimes.com/2013/08/27/business/global/milk-powder-companies-tune-in-to-chinese-socialmedia-reports.html

13. Kelly, H. (2013, October 17). Facebook changes privacy settings for teens. Retrieved from $\mathrm{http} / / / \mathrm{www} . \mathrm{cnn} . \mathrm{com} / 2013 / 10 / 16 / \mathrm{tech} / \mathrm{social}-\mathrm{media} /$ facebook-teens-privacy/

14. LiveJournal - Wikipedia, the free encyclopedia. (2013, October 22). Retrieved from http://en.wikipedia.org/wiki/LiveJournal

15. Microblogging - Wikipedia, the free encyclopedia. (2013, October 27). Retrieved from http://en.wikipedia.org/wiki/Microblogging

16. Muncaster, P. (2013, October 29). Syrian Electronic Army claims Obama social media hijacking. Retrieved from http://www.theregister.co.uk/2013/10/29/sea_hijack_obama_twitter_facebook_hack/

17. Myspace - Wikipeida, the free encyclopedia. (2013, October 21$)$. Retrieved from http://en.wikipedia.org/wiki/Myspace

18. Rouse, M. (2012, August). What is social curation? - Definition from WhatIs.com. Retrieved from $\mathrm{http}: / /$ whatis.techtarget.com/definition/social-curation

19. Rouse, M. (2012, November). What is social media? Retrieved from http://whatis.techtarget.com/definition/social-media

20. Sengupta, S. (2013, October 26). The Information-Gathering Paradox. Retrieved from 
http://www.nytimes.com/2013/10/27/sunday-review/the-information-gathering-paradox.html?src=recg

21. Siciliano, R. (2013, January 28). The Social Media Identity Theft of a School Director Via Twitter. Retrieved from http:/www.huffingtonpost.com/robert-siciliano/highschooler-goes-social-media_b_2550541.html

22. Social bookmarking - Wikipedia, the free encyclopedia. (2013, October 8). Retrieved from http://en.wikipedia.org/wiki/Social_bookmarking

23. Social networking service - Wikipedia, the free encyclopedia. (2013, October 27). Retrieved from http://en.wikipedia.org/wiki/Social_networking_service

24. The social networks of yesteryear. $\bar{H}$ ow the mighty have fallen. (2011, September 26). Retrieved from Royal Pingdom: http://royal.pingdom.com/2011/09/26/social-networks-of-yesteryear/

25. Tsukayama, H. (2013, October 11). Facebook privacy: Users should check these settings as new changes roll out. Retrieved from http://articles.washingtonpost.com/2013-10-11/business/42922974_1_facebook-privacyprivacy-tools-facebook-users

26. Usenet's Early Origins and A News. (2013). Retrieved from http://www.giganews.com/usenet-history/ origins.html

27. Vietnam court convicts dissident Facebook user. (2013, October 29). Retrieved from http://www.businessweek.com/ap/2013-10-29/vietnam-court-convicts-dissident-facebook-user

28. Wiki - Wikipedia, the free encyclopedia. (2013, October 20). Retrieved from http://en.wikipedia.org/wiki/Wiki 